\title{
RADIO MAP FRAMEWORK FOR GSM POSITIONING
}

This paper introduces radio map framework for creation of GSM (Global System for Mobile communications) network radio maps. These radio maps are structured in the way that they can be utilized later on as a data source for fingerprinting-based localization methods. Attributes of fingerprinting localization method as well as particular radio signal parameters are explained. The framework handles data measurement, data transfer and data visualization scenarios. Data are basically various radio signal parameters coupled with position coordinates. These data make up a database, which allows centralized storage and opens a path to service-oriented localization architecture in the future. Visualization provides means to verify radio map quality, distribution and density in perceptible and user friendly way as well as means to present and publish achieved results. Entire system is aimed to provide usability by multiple users and further optimization. Centralization allows easy implementation of changes and system maintenance at one place.

Keywords: Localization, fingerprinting, radio map, GSM, received signal strength.

\section{Introduction}

There was undoubtedly great progress made in the area of localization systems and services over last few years and current market provides numerous navigation and localization devices. Location based services attract more subscribers every year (see [1]) and have great potential. However, there are also drawbacks of this technological advance. Growing number of standards, technical recommendations and resource exhaustion (e.g. radio wave frequency spectrum) reduces possibilities for development of new and independent technologies.

Commonly used localization methods are based on observation of miscellaneous signal parameters. Methods used in cellular networks are Cell Identification (Cell ID), Received Signal Strength (RSS), Angle of Arrival (AoA), Time of Arrival (ToA) and Time Difference of Arrival (TDoA) [2],[3], [4] and [5]. The research of many specialists is focused on RSS method [6], [7], [8], [9] and [10], which can be then processed using trilateration technique [11], [12] or Fingerprinting technique [7], [8] and [13]. Fingerprinting seems to be most accurate and affordable technique as it is suitable for Non Line-of-Sight (NLoS) environments as well as it is more immune to multipath than trilateration.

This work is aimed to show solution that uses fingerprinting algorithm and existing technologies and combines them into radio map framework for localization in GSM network. The framework is basically a set of measurement algorithms, communication interfaces and database of various signal parameters usable by fingerprinting method to provide localization itself. Fingerprinting method is briefly described in Chapter 1.1 below. Framework architecture is described in Chapter 2.

\subsection{Fingerprinting}

Localization methods such as RSS, AoA, ToA and their mutual combinations [14] suffer from inaccuracy caused by estimation of distance between Mobile Station (MS) and Base Stations (BSs) influenced by radio channel effects such as multipath propagation or delay spread.

The fingerprinting method even benefits from aforementioned radio channel properties. It is based on unique "fingerprints" of radio signal properties measured at certain spots. Accuracy of the method in radio networks is determined by two factors [13]. Firstly, signal properties vary very much at relatively small area. For instance, in few meters range, signal from a BS can attenuate, lose or be replaced with stronger one. Secondly, these signals are relatively constant in time. It allows data gathering and their use in future. These fingerprints form the radio map shown in Fig. 1 and described below.

A disadvantage of the method is sensitivity for environment changes - movement of pedestrians and cars, construction and demolition of buildings or weather conditions - they altogether affect signal properties. It is necessary to update the map, but basically, buildings and walls affect the signal most of all and therefore update is not needed very often.

Fingerprinting method consists of two steps. At first, radio map for particular area is created. It is basically a database of spots with known position (coordinates) coupled with various signal properties, e.g. received signal level (RxLev), signal angles or propagation time. This step is called offline phase. The offline phase is to be facilitated by the radio map framework presented in this paper.

\footnotetext{
* Peter Brida, Jozef Benikovsky

Department of Telecommunications and Multimedia, Faculty of Electrical Engineering, University of Zilina, Slovakia, E-mail: peter.brida@fel.uniza.sk
} 


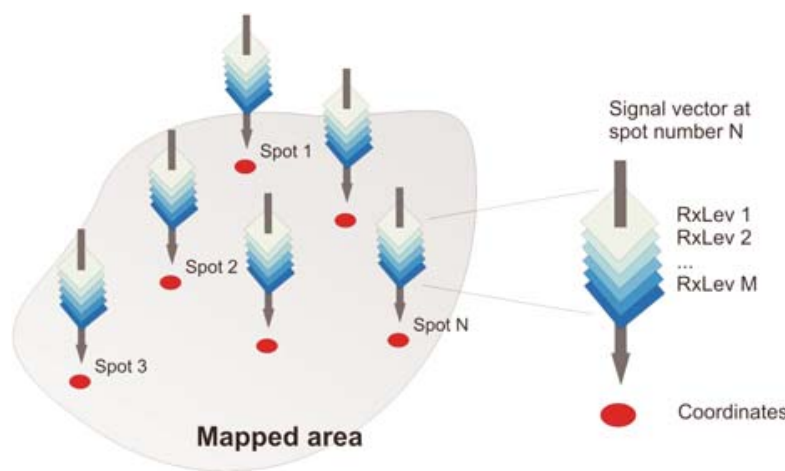

Fig. 1 Radio map for fingerprinting using RxLev

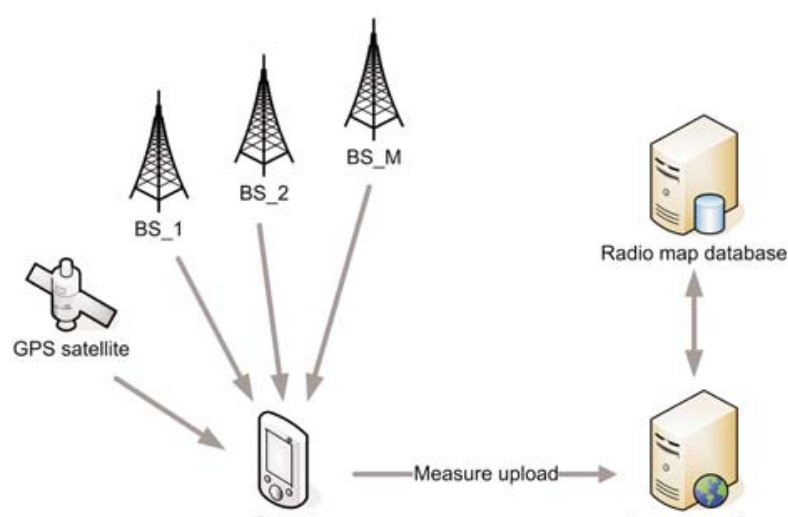

Fig. 2 Gauge subsystem
After the radio map is created, second phase can take place. MS measures signal properties at unknown spot. Then the radio map is searched to find a best match from existing spots. This step is called online phase. The online phase is not part of this paper.

\section{Radio map framework architecture}

The radio map framework consists of two parts - gauge subsystem and radio map itself [15]. Gauge subsystem is used to measure necessary signal parameters, such as identifiers of neighbour base stations coupled with received signal strength. Furthermore it measures Global Positioning System (GPS) coordinates and maps them to signal parameters. This is later user to convert signal information back into geographical coordinates. Finally, it allows transferring this data to radio map.

Radio map is the centralized storage of all measured data and gathers all essential information for fingerprinting online phase. It is actually the database, which can be accessed and queried by all clients or users.

\subsection{Gauge subsystem}

Fig. 2 shows components that make up gauge subsystem. $B S_{-} 1$, $B S \_2$ and $B S \_M$ in Fig. 2 represent GSM base transceiver stations. GPS satellite represents GPS network utilized to read "precise" position for radio map spots. Gauge is a device used to measure GSM as well as GPS signals, interpret them and transfer (upload) measurement results to communication server. The communication server then calls radio map database interfaces to append new results to database and gets notified if there were any errors.

Measurements were performed using pocket computer HP iPAQ hw6515d with Microsoft ${ }^{\circledR}$ Windows $C^{\circledR} 4.21$ and Microsoft $^{\circledR}$.NET Compact Framework 2.0 installed. This device is able to measure GPS position as well as to utilize Subscriber Identity Module Toolkit (SIM TK) to measure GSM signal para- meters. Radio map database is stored in Oracle ${ }^{\circledR}$ Express 10.0 database, which is free to use but has limited capabilities. This allows data access using standardized Structured Query Language (SQL).

\subsection{Radio map}

Radio map consists of spots. Every spot represents point in real world, where measurement was performed. Tab. 1 shows a snip of the radio map database.

Spots are uniquely identified by spot_id, which is its database identifier and is not related to GSM or GPS network. Position of the spots is defined by their position coordinates, which are latitude (in degrees), longitude (in degrees) and altitude (in meters). As shown in Tab. 1, columns with same spot_id have same position coordinates. There can be up to 7 measurements of GSM signal parameters performed for every spot, one for serving base station and one for up to six neighbour base stations. There are 3 measurements for spot 28914 and 4 measurements for spot 28915 shown in Tab. 1. Parameters measured from GSM network are:

- RxLev - received signal strength (in $\mathrm{dBm}$ ) of BS under measure,

- CI - cell identity, which is unique identifier of BS in GSM network. It is not always available, therefore $\mathrm{BCCH}$ and BSIC are measured as well,

- BCCH - Broadcast Control Channel number,

- BSIC - Base Station Identification Code. Together with BCCH uniquely identifies BS in GSM network.

As shown in Tab. 1, spots with SPOT_ID equal to 28914 and 28915 are close together (according to latitude and longitude row). It is clear that at both spots, signal from base station with $\mathrm{CID}=23342$ is received with same RxLev. However the RxLev from the subsequent base stations are different. Furthermore, number of base stations differs as well. That shows the power of fingerprinting - even for near spots, signal parameters can change significantly and localization can be performed. 


\section{COMMNICOIIONS}

Radio map database example from Zilina-Banova, Slovakia, GSM operator Orange Slovensko.

Table 1

\begin{tabular}{|l|c|c|c|c|c|c|c|}
\hline SPOT_ID & 28914 & 28914 & 28914 & 28915 & 28915 & 28915 & 28915 \\
\hline RxLev & -73 & -67 & -78 & -79 & -65 & -73 & -78 \\
\hline CID & 20872 & 24343 & 24342 & 22401 & 39893 & 20872 & 24342 \\
\hline BCCH & 3 & 13 & 8 & 10 & 20 & 3 & 8 \\
\hline BSIC & 29 & 27 & 27 & 24 & 26 & 29 & 27 \\
\hline Latitude & 49,20206 & 49,20206 & 49,20206 & 49,20204 & 49,20204 & 49,20204 & 49,20204 \\
\hline Longitude & 18,72063 & 18,72063 & 18,72063 & 18,72065 & 18,72065 & 18,72065 & 18,72065 \\
\hline Altitude & 390 & 390 & 390 & 391 & 391 & 391 & 391 \\
\hline
\end{tabular}

\subsection{Radio map visualization}

The radio map visualization provides inevitable means to get an overview of data in radio map. It is generated by web server and Google Maps ${ }^{\mathrm{TM}}$ API using connection into radio map database.

It allows displaying of all spots from radio database at once as shown in Fig. 3. Spots are shown as black dots.

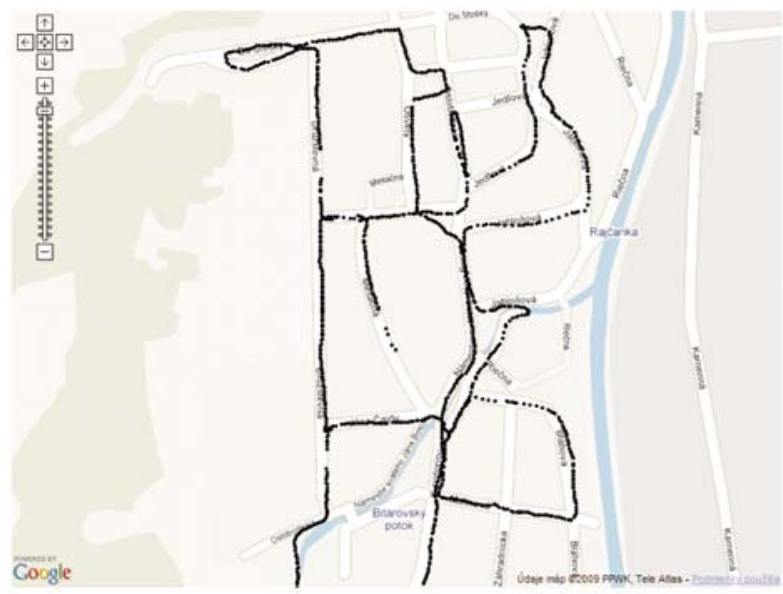

Fig. 3 Radio map database with all spots

Besides, it allows picking one BS and displaying all spots where signal of this particular BS was available as shown in Fig. 4.

\section{Future works}

Future works can be focused on radio map extrapolation, which would allow estimation of radio signal properties at unknown spots as well as comprehensive visualization options. This would also allow better accuracy in areas of radio map with weaker or missing signal information.

The development of fingerprinting online phase component would enable radio map framework to become usable service-oriented solution for localization in GSM networks.

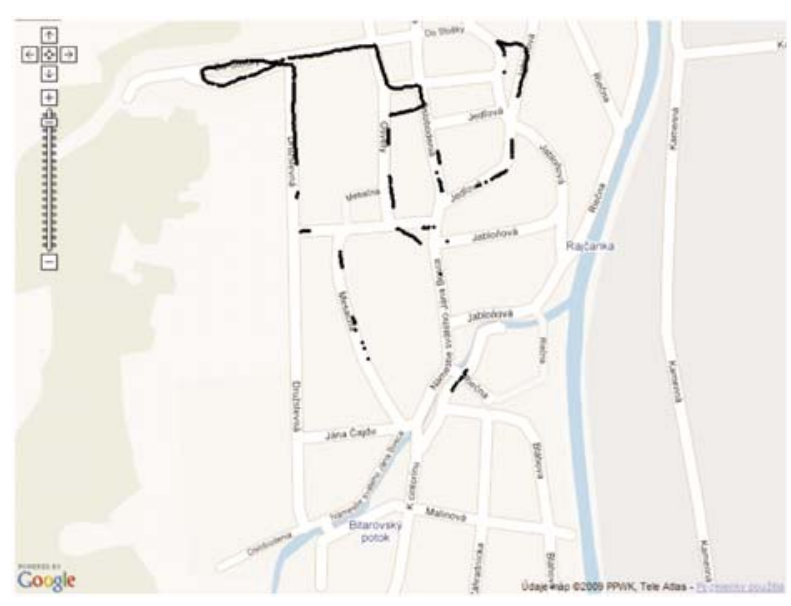

Fig. 4 Radio map database filtered for $B S$ with $C I D=4 F 81, B S I C=29$ and $B C C H=15$

For positioning in areas like buildings, where GPS signal is not available in general, extension of the framework could be proposed. It would allow measuring signal parameters without need of GPS signal. This would have allowed indoor and outdoor positioning using only one device, if localization service had been implemented.

\section{Conclusion}

Proposed solution shows combination of existing technologies to create robust framework for further localization development. It provides means to create radio map database of any area covered by GSM signal with no dependency on certain mobile network operator. By use of Oracle ${ }^{\circledR}$ Express database and corresponding SQL support, it gives a hand to advanced data research. Data visualization on the other hand provides means for user to easily understand radio map and get an overview of its current state. The core of the proposed solution is "open system" and it can be utilized for various location based services, e.g. alternative positioning solution in intelligent transport system for monitoring vehicles in dense urban areas where GPS does not reliably operate. 


\section{Acknowledgement}

The work on this paper was partially supported by the grant VEGA 1/4065/07 of Scientific Grant Agency of the Slovak Republic.

\section{References}

[1] Location Based Services Forecast from ABI Research, cit. [2008-11-12], available on internet: http://www.twine.com/item/11djpjdj2172/location-based-services-forecast-from-abi-reserach

[2] WANG X., WANG Z., O'DEA B.: A TOA-based location algorithm reducing the errors due to non-line-of-sight (NLOS) propagation. IEEE Transactions on Vehicular Technology, 2003, Vol. 52, No. 1, pp. 112-116.

[3] NEMEC Z., BEZOUSEK P., The Time Difference of Arrival Estimation of Wi-Fi Signals. Radioengineering, Vol. 17, No. 4, pp. 51-54, ISSN: 1210-2512, 2008.

[4] DELIGIANNIS N., KOTSOPOUlOS S., Mobile Positioning Based on Existing Signalling Messaging in GSM Networks. In Proceedings of 3rd International Mobile Multimedia Communications Conference (MSAN), 27-29 August 2007, Greece: Nafpaktos.

[5] BRIDA P., CEPEL P., DUHA J., Mobile Positioning in Next Generation Networks. (Chapter XI), in Kotsopoulos, S. \& Ioannou, K. (Ed.), Handbook of Research on Heterogeneous Next Generation Networking: Innovations and Platforms (pp. 223-252). New York, Hershey: IGI Global (Information science reference), 10/2008. ISBN 978-1-60566-108-7.

[6] BRIDA P.,CEPEL P., DUHA J., "A Novel Adaptive Algorithm for RSS Positioning in GSM Networks", in CSNDSP 2006 PROCEEDINGS, ISBN 960-89282-0-6. Patras, Greece, 19-21 July, 2006, pp. 748-751.

[7] ANNE K. R., KYAMAKYA K., ERBAS F., TAKENGA C., CHEDJOU J. C., GSM RSSI-based Positioning Using Extended Kalman Filter for Training ANN. IEEE Vehicular Technology Conference No. 60, Los Angeles, USA, 2004, Vol. 7, pp. 4141-4145, ISBN: 0-7803-8521-7.

[8] LAITINEN H., NORDSTROM T., LAHTEENMAKI J., Location of GSM Terminals Using a Database of Signal Strength Measurements. in URSI XXV, National Convention on Radio Science, Helsinki, September 2000.

[9] TAKENGA C., KYAMAKYA K., QUAN W., On the Accuracy Improvement Issues in GSM Location Fingerprinting. Vehicular Technology Conference, 2006, VTC-2006 Fall, ISBN: 1-4244-0062-7, Hannover 25-28 Sept. 2006, pp. 1-5.

[10] NERGUIZIAN C., DESPINS C., AFFES S., Indoor Geolocation with Received Signal Strength Fingerprinting Technique and Neural Networks. J. N. de Souza et al. (Eds.): ICT 2004, LNCS 3124, 2004, pp. 866-875, ISBN: 978-3-540-22571-3.

[11] M. HATA, T. NAGATSU, Mobile Location Using Signal Strength Measurements in a Cellular System. IEEE Trans. On Vehicular Technology, May 1980, Vol. VT-29, pp. 245-252.

[12] WONG C. L. C., LEE M. C., CHAN R. K. W., GSM-based mobile positioning using WAP. Wireless Communications and Networking Conference. WCNC. 2000 IEEE, September 2000, Vol. 2, pp. 874-878.

[13] OTSASON, V.: Accurate Indoor Localization Using Wide GSM Fingerprinting, Master's thesis, Tartu, 2005.

[14] BENIKOVSKY, J.: Creation of System for Localization in Real GSM Network (in Slovak), Master's thesis, Zilina, 2008.

[15] BENIKOVSKY, J., BRIDA, P.: Positioning System Based on Fingerprinting Method for Mobile Cellular Networks. The Mediterranean Journal of Computers and Networks, Vol. 5, 2/2009, pp. 59-67, ISSN: 1744-2397. 